\title{
GEOMETRIC INVARIANTS FOR SEIFERT FIBRED 3-MANIFOLDS
}

\author{
MINGQING OUYANG
}

\begin{abstract}
In this paper, we obtain a formula for the $\eta$-invariant of the signature operator for some circle bundles over Riemannian 2-orbifolds. We then apply it to Seifert fibred 3-manifolds endowed with one of the six Seifert geometries. By using a relation between the Chern-Simons invariant and the $\eta$-invariant, we also derive some elementary formulae for the Chern-Simons invariant of these manifolds. As applications, we show that some families of these manifolds cannot be conformally immersed into the Euclidean space $\mathbf{E}^{4}$.
\end{abstract}

\section{INTRODUCTION}

In dimension 3, the work of Thurston [15], [16] indicates that there are essentially eight relevant homogeneous geometries needed for geometric structures on 3-manifolds. Of these, six are the so-called Seifert geometries.

The purpose of this paper is to study two kinds of geometric invariants, the Chern-Simons invariant and the $\eta$-invariant, for a 3-manifold endowed with one of these six Seifert geometries.

The Chern-Simons invariant was first defined in [3] for a closed Riemannian 3-manifold by integrating a certain 3-form over the manifold. It is only defined $\bmod \mathbf{Z}$.

The $\eta$-invariant of a selfadjoint elliptic operator was originally introduced by Atiyah, Patodi, and Singer in [1] for odd-dimensional Riemannian manifolds in terms of the spectrum of the operator. The $\eta$-invariant we are interested in here is that of the signature operator. Such an invariant has a topological interpretation which allows us to compute it without using analytic tools. As shown in [1], it measures the extent to which the Hirzebruch signature formula fails for a nonclosed $4 k$-dimensional Riemannian manifold whose metric is a product near its boundary. In dimension 3, it can be thought of as a real-valued generalization of the Chern-Simons invariant.

The remainder of this paper consists of three sections. In Section 2, we obtain a formula for the $\eta$-invariant of $S^{1}$-bundles over Riemannian 2-orbifolds. We then, in Section 3, apply our formula to the geometric Seifert fibred 3-manifolds to get explicit expressions of the $\eta$-invariant under these geometries. Finally, in Section 4, we derive some elementary formulae for the Chern-Simons invariant of geometric Seifert fibred 3-manifolds. As applications, we show that some

Received by the editors September 17, 1993 and, in revised form, January 26, 1994 and March 10, 1994.

1991 Mathematics Subject Classification. Primary 57M50, 53C30; Secondary 58G25, 58C40. 
families of these manifolds cannot be conformally immersed into the Euclidean space $\mathbf{E}^{4}$.

\section{2. $\eta$-INVARIANT FOR $S^{1}$-BUNDLES OVER RIEMANNIAN 2-ORBIFOLDS}

In [10], Komuro obtained a formula for the $\eta$-invariant of some circle bundles over Riemann surfaces. In this section, we extend his formula to the case of $S^{1}$-bundles over Riemannian 2-orbifolds by using the generalized Hirzebruch signature formula as developed by Kawasaki in [8] and [9]. We start with the following topological interpretation for the $\eta$-invariant due to Atiyah, Patodi, and Singer:

Theorem 2.0 [1]. Let $N$ be a $4 k$-dimensional Riemannian manifold with $\partial N=$ $M$. Assume that $N$ has a product metric near the boundary. Then

$$
\eta(M)=\int_{N} L_{k}(P)-\operatorname{Sign}(N)
$$

where $L_{k}$ is the kth Hirzebruch L-polynomial, $P$ is the Pontrjagin form of $N$ and $\operatorname{Sign}(N)$ is the signature of $N$.

Kawasaki in [8] generalized the above theorem to the case where $M^{4 k-1}$ bounds a $4 k$-dimensional Riemannian orbifold.

To state his result, we need some preliminaries from [8] and [9].

Let $(X, \mathscr{U})$ be a compact orbifold. Denote by $\Sigma X$ the singular set of $X$. For every $x \in \Sigma X$, choose precisely one linear chart $\left(\tilde{U}_{x}, G_{x}, U_{x}, \pi_{x}\right)$ such that $\pi_{x}(0)=x$. For each $g \in G_{x}$, a local group of $x$, the centralizer $C_{G_{x}}(g)$ of $g$ in $G_{x}$ acts on $\tilde{U}_{x}^{g}=\left\{y \in \tilde{U}_{x} \mid g \cdot y=y\right\}$. Let $(1),\left(h_{x}^{1}\right), \cdots,\left(h_{x}^{\rho_{x}}\right)$ be all the conjugacy classes in $G_{x}$. Suppose $y \in U_{x} \cap \Sigma X$. Then an overlap map $\psi_{y x}$ between $\tilde{U}_{y}$ and $\tilde{U}_{x}$ induces a homomorphism $\psi_{y x}^{\star}: G_{y} \longrightarrow G_{x}$. The following is a bijection

$$
\begin{aligned}
\left\{\left(y,\left(h_{y}^{j}\right)\right) \mid y \in U_{x} \cap \Sigma X, \psi_{y x}^{\star}\left(h_{y}^{j}\right)=h_{x}^{i}\right\} & \longrightarrow \tilde{U}_{x}^{h_{x}^{i}} / C_{G_{x}}\left(h_{x}^{i}\right), \\
\left(y,\left(h_{y}^{j}\right)\right) & \longmapsto\left[\psi_{y x}(0)\right]
\end{aligned}
$$

which defines an orbifold structure on

$$
\tilde{\Sigma} X=\left\{\left(x,\left(h_{x}^{j}\right)\right) \mid x \in \Sigma X, j=1,2, \cdots, \rho_{x}\right\} .
$$

The multiplicity of $\tilde{\Sigma} X$ in $X$ at $\left(x,\left(h_{x}^{j}\right)\right)$ is the order of the trivially acting subgroup of $C_{G_{x}}\left(h_{x}^{j}\right)$ on $\tilde{U}_{x}^{h_{x}^{j}}$.

Let $\tilde{\Sigma}_{1} X, \ldots, \tilde{\Sigma}_{c} X$ be the connected components of $\tilde{\Sigma} X$. The multiplicity is locally constant on $\tilde{\Sigma} X$. Thus we can assign a number $m_{s}=m\left(\tilde{\Sigma}_{s} X\right)$ to each $\tilde{\Sigma}_{s} X$, called the multiplicity of $\tilde{\Sigma}_{s} X$ in $X$.

On each orbifold chart $\tilde{U}_{x}^{h}$ of $\tilde{\Sigma} X$, we have the normal bundle $\nu\left(\tilde{U}_{x}^{h}\right)$ in $\tilde{U}_{x}$ and the tangent bundle $\tau\left(\tilde{U}_{x}^{h}\right) . h$ acts on $\nu\left(\tilde{U}_{x}^{h}\right)$. We have the decomposition

$$
\nu\left(\tilde{U}_{x}^{h}\right)=\bigoplus_{0<\theta \leq \pi} \nu_{\theta}^{h}
$$

where $\nu_{\theta}^{h}$ is the bundles of eigenspace for $h$ with eigenvalues $e^{ \pm i \theta}$. Introduce complex structures on the bundles $\nu_{\theta}^{h}$ such that $h \cdot v=e^{i \theta} v$ if $v \in \nu_{\theta}^{h}$. (For 
simplicity, we assume here that $\nu_{\pi}^{h}$ can be given a compatible complex structure so that $\theta=\pi$ need not play a special role). The collection of these $C_{G_{x}}(h)$ bundles form complex vector bundles over $\tilde{\Sigma} X$. Choose a $C_{G_{x}}(h)$-invariant connection for each bundle. Write the total Chern class

$$
c\left(\nu_{\theta}^{h}\right)=\prod_{j}\left(1+x_{j}\right),
$$

i.e., $c_{n}\left(\nu_{\theta}^{h}\right)=n$th symmmetric polynomial in $x_{j}$ 's. Define

$$
L_{\theta}\left(\nu_{\theta}^{h}\right)=\prod_{j} \operatorname{coth}\left(x_{j}+\frac{i \theta}{2}\right)=\prod_{j}\left(\frac{e^{i \theta} e^{2 x_{j}}+1}{e^{i \theta} e^{2 x_{j}}-1}\right) .
$$

The local characteristic form

$$
L^{\tilde{\Sigma}}\left(\tilde{U}_{x}^{h}\right)=L\left(\tilde{U}_{x}^{h}\right) \cdot \prod_{0<\theta \leq \pi} L_{\theta}\left(\nu_{\theta}^{h}\right)
$$

defines an $L$-class in $\tilde{\Sigma} X$.

Theorem 2.1 (Kawasaki [8]). Let $N$ be a $4 k$-dimensional Riemannian orbifold with $\partial N=M a(4 k-1)$-dimensional Riemannian manifold. Assume that $N$ has a product metric near the boundary. Then

$$
\eta(M)=\int_{N} L_{k}(P)-\operatorname{Sign}(N)+\sum_{s=1}^{c} \frac{1}{m_{s}}\left\langle L^{\tilde{\Sigma}_{s}},\left[\tilde{\Sigma}_{s} N\right]\right\rangle .
$$

In Theorems 2.0 and 2.1, it is required that the metric on $N$ is a product near the boundary. But this may not be the case in practice. We need a so-called "boundary correction term". The following formulation is due to Gilkey [4].

Let $C=M \times[0,1]$ and identify $M \times\{0\}$ with $M=\partial N$. Let $g_{0}$ be the product metric on $C$. Extend the metric $g$ on $N$ to a metric $g_{1}$ on $C \cup N$ such that $g_{1}$ is a product near $M \times\{1\}$ and agrees with $g_{0}$ near $M \times 1$. Let $\nabla_{0}$ and $\nabla_{1}$ be the Riemannian connections determined by $g_{0}$ and $g_{1}$ respectively. Denote $\omega_{0}, \omega_{1}$ and $\Omega_{0}, \Omega_{1}$ the corresponding connection forms and curvature forms of $\nabla_{0}$ and $\nabla_{1}$ respectively.

Let $\omega=\omega_{1}-\omega_{0}, \omega_{t}=(1-t) \omega_{0}+t \omega_{1}$, and $\Omega_{t}$ the curvature form of $\omega_{t}$.

Define

$$
T L_{k}=2 k \int_{0}^{1} L_{k}\left(\omega, \Omega_{t}, \cdots, \Omega_{t}\right) d t .
$$

Then

$$
d T L_{k}=L_{k}\left(\Omega_{1}\right)-L_{k}\left(\Omega_{0}\right) .
$$

Since $L_{k}\left(\Omega_{0}\right)=0$, we get

$$
d T L_{k}=L_{k}\left(\Omega_{1}\right)=L_{k}\left(\Omega_{1}, \cdots, \Omega_{1}\right) .
$$

Thus

$$
\int_{C} L_{k}\left(\Omega_{1}\right)=\int_{C} d T L_{k}=\int_{\partial C} T L_{k}=\int_{M \times\{0\}} T L_{k}=-\int_{M} T L_{k} .
$$


It follows from Theorem 2.1 that

$$
\begin{aligned}
\eta(M) & =\int_{C \cup N} L_{k}(P)-\operatorname{Sign}(C \cup N)+\sum_{s=1}^{c} \frac{1}{m_{s}}\left\langle L^{\tilde{\Sigma}_{s}},\left[\tilde{\Sigma}_{s} N\right]\right\rangle \\
& =\int_{C} L_{k}\left(\Omega_{1}\right)+\int_{N} L_{k}(P)-\operatorname{Sign}(N)+\sum_{s=1}^{c} \frac{1}{m_{s}}\left\langle L^{\tilde{\Sigma}_{s}},\left[\tilde{\Sigma}_{s} N\right]\right\rangle \\
& =-\int_{M} T L_{k}+\int_{N} L_{k}(P)-\operatorname{Sign}(N)+\sum_{s=1}^{c} \frac{1}{m_{s}}\left\langle L^{\tilde{\Sigma}_{s}},\left[\tilde{\Sigma}_{s} N\right]\right\rangle .
\end{aligned}
$$

Now, let $S(E) \longrightarrow F$ be a principal $S^{1}$-bundle over an oriented, Riemannian 2-orbifold $(F, \hat{g})$. Let $p: E \longrightarrow F$ be the associated $\mathbf{C}^{1}$-bundle.

Choose a fiber metric $\tilde{g}$ and a $\tilde{g}$-preserving connection $\tilde{\nabla}$. Then we obtain a Riemannian orbifold $(E, g)$ by assuming that

$$
\left.g\right|_{\text {horizontal }}=p^{\star} \hat{g} \quad \text { and }\left.\quad g\right|_{\text {vertical }}=\tilde{g} \text {. }
$$

We now determine the Riemannian connection of $g$ on $E$.

For any $x \in F$, choose local orthonormal (horizontal) sections $e_{1}, e_{2}$ near $x$, Then they determine a coordinate system $u^{1}, u^{2}$ along the fiber with $e_{\alpha}=$ $\partial / \partial u^{\alpha}, \alpha=1,2$.

Let $x_{3}, x_{4}$ be a local orthonormal frame of $F$ near $x$ and $e_{3}, e_{4}$ their horizontal lifts with respect to $\widetilde{\nabla}$.

Let $\tilde{R}$ be the curvature tensor of the connection $\tilde{\nabla}$ and $\tilde{\Omega}_{\alpha}^{\beta}$ its components with respect to $e_{1}, e_{2}$. Then $\tilde{\Omega}_{1}^{2}=\tilde{R}_{1234} x_{3}^{\star} \wedge x_{4}^{\star}$ where

$$
\tilde{R}_{1234}=\tilde{g}\left(\tilde{R}\left(x_{3}, x_{4}\right) e_{1}, e_{2}\right)
$$

We will denote $\tilde{R}=\tilde{R}_{1234}$ for brevity. For convenience, we use the polar coordinate system $(r, \theta)$ in the vertical space so that $u^{1}=r \cos \theta$ and $u^{2}=$ $r \sin \theta$.

A straightforward calculation yields

Lemma 2.2. With respect to the basis $\left\{e_{1}=\frac{1}{r} \frac{\partial}{\partial \theta}, e_{2}=\frac{\partial}{\partial r}, e_{3}, e_{4}\right\}$, the connection form $\omega$ of $\nabla$ is given by

$$
\begin{aligned}
& \omega_{2}^{1}=\widetilde{d \theta}, \quad \omega_{3}^{1}=-\frac{1}{2} r \tilde{R} e_{4}^{\star}, \quad \omega_{4}^{1}=\frac{1}{2} r \tilde{R} e_{3}^{\star}, \\
& \omega_{3}^{2}=\omega_{4}^{2}=0, \quad \omega_{4}^{3}=-\frac{1}{2} r^{2} \tilde{R} \widetilde{d \theta}+p^{\star} \hat{\omega}_{4}^{3}
\end{aligned}
$$

where $\left\{r \widetilde{d \theta}, d r, e_{3}^{\star}, e_{4}^{\star}\right\}$ is the dual basis of $\left\{\frac{1}{r} \frac{\partial}{\partial \theta}, \frac{\partial}{\partial r}, e_{3}, e_{4}\right\}$ and $\hat{\omega}_{4}^{3}$ is the component of the connection form of $\hat{g}$ with respect to $x_{3}, x_{4}$.

From Lemma 2.2, we have the following 
Lemma 2.3. If $\tilde{R}$ is constant on $F$, then with respect to the same basis as in Lemma 2.2, the components of the curvature form $\Omega$ of $\nabla$ are given by

$$
\begin{aligned}
& \Omega_{1}^{2}=\tilde{R} e_{3}^{\star} \wedge e_{4}^{\star}, \\
& \Omega_{1}^{3}=-\frac{1}{2} \tilde{R} e_{4}^{\star} \wedge d r+\frac{1}{4} r^{2} \tilde{R}^{2} e_{3}^{\star} \wedge r \widetilde{d \theta}, \\
& \Omega_{1}^{4}=\frac{1}{2} \tilde{R} e_{3}^{\star} \wedge d r+\frac{1}{4} r^{2} \tilde{R}^{2} e_{4}^{\star} \wedge r \widetilde{d \theta}, \\
& \Omega_{2}^{3}=\frac{1}{2} \tilde{R} e_{4}^{\star} \wedge r \widetilde{d \theta}, \\
& \Omega_{2}^{4}=-\frac{1}{2} \tilde{R} e_{3}^{\star} \wedge r \widetilde{d \theta}, \\
& \Omega_{4}^{3}=p^{\star} \hat{\Omega}_{3}^{4}-\frac{3}{4} r^{2} \tilde{R}^{2} p^{\star} \omega_{F}+\tilde{R} d r \wedge r \widetilde{d \theta},
\end{aligned}
$$

where $\hat{\Omega}_{3}^{4}$ is the curvature form of $\hat{\omega}_{3}^{4}$ and $\omega_{F}=x_{3}^{\star} \wedge x_{4}^{\star}$ is the volume form of $(F, \hat{g})$. Equation (2) is valid in general.

Before presenting our main theorem, we need some facts about the local structure of a 2-orbifold and a complex line bundle over it.

Let $F$ be an oriented, closed 2-orbifold. Then, by the classification theorem of 2-orbifolds (see [15], for example), the only possible orbifold charts have the form $\mathbf{R}^{2} /(\mathbf{Z} / \alpha)$ with $\mathbf{Z} / \alpha$ acting on $\mathbf{R}^{2}$ by multiplication by $e^{2 \pi \beta i / \alpha}$ for some $\beta$ prime to $\alpha$. $\Sigma F$ consists of a set of isolated points $\left\{x_{1}, \cdots, x_{n}\right\}$ in $F$. Thus, $F$ is determined by the data $\left(g ; \alpha_{1}, \cdots, \alpha_{n}\right)$ where $g$ is the genus of $F$ and the $\alpha_{s}$ 's are such that $G_{x_{s}}=\mathbf{Z} / \alpha_{s}$. We assume thereafter, without loss of generality, that $\alpha_{s}>0$.

Let $E \longrightarrow F$ be a complex line bundle over $F$ such that $E$ is a 4-orbifold with orbifold chart $\left(\mathbf{R}^{2} \times \mathbf{R}^{2}\right) / \mathbf{Z} / \alpha$ where $\mathbf{Z} / \alpha$ acts on the first coordinate by $e^{2 \pi i \beta / \alpha}$ and on the second by $e^{2 \pi \gamma i / \alpha}$ for some $\beta$ and $\gamma$ prime to $\alpha$. Thus $\Sigma E=\left\{\left(x_{1}, 0\right), \cdots,\left(x_{n}, 0\right)\right\}$. We call

$$
\left(\left(\alpha_{1} ; \beta_{1}, \gamma_{1}\right), \cdots,\left(\alpha_{n} ; \beta_{n}, \gamma_{n}\right)\right)
$$

the orbifold data of $E$.

Theorem 2.4. Let $p: E \longrightarrow F$ be a complex line bundle over an oriented, closed Riemannian 2-orbifold $(F, \hat{g})$. Suppose that the total space $E$ has orbifold data $\left(\left(\alpha_{1} ; \beta_{1}, \gamma_{1}\right), \cdots,\left(\alpha_{n} ; \beta_{n}, \gamma_{n}\right)\right)$. Choose a fiber metric $\tilde{g}$ in $E$ and let $\tilde{\nabla}$ be a $\tilde{g}$-preserving connection in $E$. Then $(E, g)$ becomes a Riemannian orbifold. Assume that $\tilde{R}$ is constant on $F$. Then the $\eta$-invariant of the circle bundle of radius $r$ is given by

$$
\eta\left(S_{r} E\right)=\frac{2}{3} c_{1}\left\{\frac{\pi r^{2}}{\operatorname{Vol}(F)} \chi-\left(\frac{\pi r^{2}}{\operatorname{Vol}(F)}\right)^{2} c_{1}^{2}\right\}+\frac{1}{3} c_{1}-\varepsilon+\sum_{j=1}^{n} 4 s\left(\beta_{j}, \gamma_{j} ; \alpha_{j}\right)
$$

where $c_{1}$ is the (rational) Euler number of the bundle $E \longrightarrow F . \chi$ is the (rational) Euler characteristic of the base orbifold $F . s\left(\beta_{j}, \gamma_{j} ; \alpha_{j}\right)$ is the following 
generalized Dedekind sum as in [7]:

$$
s(\beta, \gamma ; \alpha)=\frac{1}{4 \alpha} \sum_{k=1}^{\alpha-1} \cot \left(\frac{k \beta \pi}{\alpha}\right) \cot \left(\frac{k \gamma \pi}{\alpha}\right) .
$$

$\varepsilon$ is defined by

$$
\varepsilon= \begin{cases}1 & \text { if } c_{1}>0 \\ 0 & \text { if } c_{1}=0 \\ -1 & \text { if } c_{1}<0\end{cases}
$$

Proof. Denote by $D_{r}(E)$ the disk-bundle of radius $r$ of $E$. Let $g_{0}=\left.g\right|_{S_{r}(E)}$ $\times d t^{2}$ be the product metric on $D_{r+1}(E)-D_{r}(E)=S_{r}(E) \times[0,1]$. Let $\nabla_{0}$ be the Riemannian connection determined by $g_{0}$ and $\omega_{0}, \Omega_{0}$ the connection form and curvature form of $\nabla_{0}$ respectively. Choose a metric $g_{1}$ on $D_{r+1}(E)-$ $D_{r}(E)$ such that $g_{1}=g$ on $D_{r+\frac{1}{4}}(E)-D_{r}(E)$ and $g_{1}=g_{0}$ on $D_{r+1}(E)-$ $D_{r+\frac{3}{4}}(E)$. Let $\nabla_{1}$ be the Riemannian connection determined by the metric $g_{1}$ and $\omega_{1}, \Omega_{1}$ the connection form and curvature form of $\nabla_{1}$ respectively.

Write $\omega=\omega_{1}-\omega_{0}$ and $\omega_{t}=(1-t) \omega_{0}+t \omega_{1}$. Then from Lemma 2.2, with respect to the basis $\left\{\frac{1}{r} \frac{\partial}{\partial \theta}, \frac{\partial}{\partial r}, e_{3}, e_{4}\right\}$, the components of $\omega_{t}$ on $D_{r+\frac{1}{4}}(E)-$ $D_{r}(E)$ are given by

$$
\begin{aligned}
& \left(\omega_{t}\right)_{2}^{1}=t \widetilde{d \theta}, \quad\left(\omega_{t}\right)_{3}^{1}=-\frac{1}{2} r \tilde{R} e_{4}^{\star}, \quad\left(\omega_{t}\right)_{4}^{1}=\frac{1}{2} r \tilde{R} e_{3}^{\star}, \\
& \left(\omega_{t}\right)_{3}^{2}=\left(\omega_{t}\right)_{4}^{2}=0, \quad\left(\omega_{t}\right)_{4}^{3}=-\frac{1}{2} r^{2} \tilde{R} \widetilde{d \theta}+p^{\star} \hat{\omega}_{4}^{3} .
\end{aligned}
$$

It follows that $\left(\Omega_{t}\right)_{1}^{2}=t p^{\star} \tilde{\Omega}_{1}^{2}$. Also, $\omega_{1}^{2}=-\widetilde{d \theta}$ and $\omega_{s}^{q}=0$ others. From Lemma 2.3 we get

$$
\begin{aligned}
& \left(\Omega_{1}^{2}\right)^{2}=\left(\Omega_{2}^{3}\right)^{2}=\left(\Omega_{2}^{4}\right)^{2}=0 \\
& \left(\Omega_{1}^{3}\right)^{2}+\left(\Omega_{1}^{4}\right)^{2}=-\frac{1}{2} r^{2} \tilde{R}^{3} e_{3}^{\star} \wedge e_{4}^{\star} \wedge r d r \wedge \widetilde{d \theta} \\
& \left(\Omega_{3}^{4}\right)^{2}=-\frac{3}{2} r^{2} \tilde{R}^{3} e_{3}^{\star} \wedge e_{4}^{\star} \wedge r d r \wedge \widetilde{d \theta}+2 p^{\star} \hat{\Omega}_{3}^{4} \wedge \tilde{R} d r \wedge r \widetilde{d \theta}
\end{aligned}
$$

Thus,

$$
\begin{aligned}
& L_{1}(g)=\frac{1}{3} P_{1}(g)=\frac{1}{12 \pi^{2}} \sum_{s<q}\left(\Omega_{s}^{q}\right)^{2} \\
&=\frac{1}{6 \pi^{2}}\left(\tilde{R} p^{\star} \hat{\Omega}_{3}^{4} \wedge r d r \wedge \widetilde{d \theta}-r^{2} \tilde{R}^{3} p^{\star} \omega_{F} \wedge r d r \wedge \widetilde{d \theta}\right), \\
& T L_{1}=2 \int_{0}^{1} L_{1}\left(\omega, \Omega_{t}\right) d t \\
&=2 \cdot \frac{1}{12 \pi^{2}} \int_{0}^{1} t p^{\star} \tilde{\Omega}_{1}^{2} \wedge(-\widetilde{d \theta}) d t \\
&=-\frac{1}{12 \pi^{2}} p^{\star} \tilde{\Omega}_{1}^{2} \wedge \widetilde{d \theta}
\end{aligned}
$$


Also, note that $\hat{\Omega}_{3}^{4}$ and $\tilde{\Omega}_{1}^{2}$ represent $2 \pi \chi(F)$ and $2 \pi c_{1}(E)$ respectively. Thus, since $\tilde{R}$ is constant on $F$, we have

$$
\tilde{R}=\frac{2 \pi c_{1}}{\operatorname{Vol}(F)} .
$$

Choose a partition of unity $\left\{f_{\alpha}\right\}_{\alpha \in \Lambda}$ subordinate to the open cover $\mathscr{U}$ of $F$. Then we get

$$
\begin{aligned}
& \int_{D_{r}(E)} L_{1}(g)=\sum_{\alpha \in \Lambda} \frac{1}{\left|G_{\alpha}\right|} \int_{\tilde{E}_{\alpha}}\left(f_{\alpha} \circ p\right) \frac{1}{6 \pi^{2}}\left(\tilde{R} p^{\star} \hat{\Omega}_{3}^{4} \wedge r d r \wedge \widetilde{d \theta}\right. \\
& \left.-r^{2} \tilde{R}^{3} p^{\star} \omega_{F} \wedge r d r \wedge \widetilde{d \theta}\right) \\
& =\sum_{\alpha \in \Lambda} \frac{1}{\left|G_{\alpha}\right|} \int_{\tilde{U}_{\alpha}} f_{\alpha} \frac{1}{6 \pi^{2}}\left(\pi r^{2} \tilde{R} \hat{\Omega}_{3}^{4}-\frac{1}{2} \pi r^{4} \tilde{R}^{2} \tilde{\Omega}_{1}^{2}\right) \\
& =\int_{F} \frac{1}{6 \pi^{2}}\left(\pi r^{2} \tilde{R} \hat{\Omega}_{3}^{4}-\frac{1}{2} \pi r^{4} \tilde{R}^{2} \tilde{\Omega}_{1}^{2}\right) \\
& =\frac{1}{6 \pi^{2}}\left(\pi r^{2} \tilde{R} \hat{\Omega}_{3}^{4}-\frac{1}{2} \pi r^{4} \tilde{R}^{2} \tilde{\Omega}_{1}^{2}\right)[F] \\
& =\frac{2}{3} c_{1}\left(\frac{\pi r^{2}}{\operatorname{Vol}(F)} \chi-\left(\frac{\pi r^{2}}{\operatorname{Vol}(F)}\right)^{2} c_{1}^{2}\right) \text {, } \\
& \int_{S_{r}(E)} T L_{1}(g)=-\sum_{\alpha \in \Lambda} \frac{1}{\left|G_{\alpha}\right|} \int_{\tilde{U}_{\alpha} \times S^{1}}\left(f_{\alpha} \circ p\right) \frac{1}{12 \pi^{2}}\left(p^{\star} \tilde{\Omega}_{1}^{2} \wedge \widetilde{d \theta}\right) \\
& =-\sum_{\alpha \in \Lambda} \frac{2 \pi}{\left|G_{\alpha}\right|} \int_{\tilde{U}_{\alpha}} f_{\alpha} \frac{1}{12 \pi^{2}} \tilde{\Omega}_{1}^{2}=-\frac{1}{6 \pi} \int_{F} \tilde{\Omega}_{1}^{2} \\
& =-\frac{1}{6 \pi} 2 \pi c_{1}(E)[F]=-\frac{1}{3} c_{1} \text {. }
\end{aligned}
$$

To determine $\operatorname{Sign}\left(D_{r}(E)\right)$, we examine the following diagram:

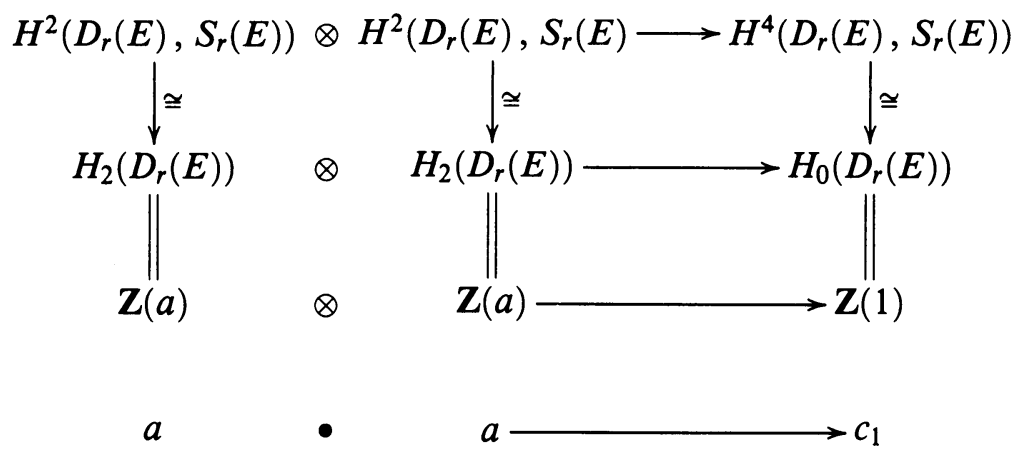

where the vertical arrows are Poincaré-Lefschetz duality with $\mathbf{Q}$ coefficients and the last row is an orbifold version of the Euler characteristic of $E$ (see 
[11], for instance). Thus, we get

$$
\operatorname{Sign}\left(D_{r}(E)\right)=\varepsilon= \begin{cases}1 & \text { if } c_{1}>0 \\ 0 & \text { if } c_{1}=0 \\ -1 & \text { if } c_{1}<0\end{cases}
$$

Next, we determine the term

$$
\sum \frac{1}{m_{s}}\left\langle L^{\Sigma_{s}},\left[\tilde{\Sigma}_{s} D_{r}(E)\right]\right\rangle
$$

By the orbifold data, we have

$$
\tilde{\Sigma} D_{r}(E)=\left\{\left(x_{j},\left(g_{j}^{k}\right)\right) \mid j=1,2, \cdots, n, k=1,2, \cdots, \alpha_{j}-1\right\}
$$

and

$$
m_{j}^{k}=\alpha_{j} \text { for } k=1,2, \cdots, \alpha_{j}-2
$$

Then,

$$
\nu_{\left(x_{j},\left(g_{j}^{k}\right)\right)}=T_{\left(x_{j},\left(g_{j}^{k}\right)\right)} E=\mathbf{C}_{1} \oplus \mathbf{C}_{2}
$$

with $g_{j}^{k}$ acting on $\mathbf{C}_{1}$ and $\mathbf{C}_{2}$ by multiplication by $e^{2 \pi \beta_{j} k i / \alpha_{j}}$ and $e^{2 \pi \gamma_{j} k i / \alpha_{j}}$ respectively. It follows that

$$
\begin{aligned}
\left\langle L^{\Sigma},\left[\tilde{\Sigma}_{j}^{k} D_{r}(E)\right]\right\rangle & =\prod_{0<\theta \leq \pi} L_{\theta}\left(\nu_{\left(x_{j},\left(g_{j}^{k}\right)\right.}^{g^{k}}\right)\left[\left(x_{j},\left(g_{j}^{k}\right)\right)\right] \\
& =\frac{e^{2 \pi \beta_{j} k i / \alpha_{j}}+1}{e^{2 \pi \beta_{j} k i / \alpha_{j}}-1} \cdot \frac{e^{2 \pi \gamma_{j} k i / \alpha_{j}}+1}{e^{2 \pi \gamma_{j} k i / \alpha_{j}}-1} \\
& =-\cot \frac{k \beta_{j} \pi}{\alpha_{j}} \cot \frac{k \gamma_{j} \pi}{\alpha_{j}} .
\end{aligned}
$$

Therefore,

$$
\begin{aligned}
\sum \frac{1}{m_{s}}\left\langle L^{\Sigma_{s}},\left[\tilde{\Sigma}_{s} D_{r}(E)\right]\right\rangle & =-\sum_{j=1}^{n} \frac{1}{\alpha_{j}} \sum_{k=1}^{\alpha_{j}-1} \cot \frac{k \beta_{j} \pi}{\alpha_{j}} \cot \frac{k \gamma_{j} \pi}{\alpha_{j}} \\
& =\sum_{j=1}^{n} \frac{1}{\alpha_{j}}\left(4 \alpha_{j} s\left(\beta_{j}, \gamma_{j} ; \alpha_{j}\right)\right) \\
& =4 \sum_{j=1}^{n} s\left(\beta_{j}, \gamma_{j} ; \alpha_{j}\right) .
\end{aligned}
$$

Finally, from formula (2.1) we get

$$
\begin{aligned}
\eta\left(S_{r} E\right) & =\int_{D_{r}(E)} L_{1}-\int_{S_{r}(E)} T L_{1}-\operatorname{Sign}\left(D_{r}(E)\right)+\sum \frac{1}{m_{s}}\left\langle L^{\tilde{\Sigma}_{s}},\left[\tilde{\Sigma}_{s} D_{r}(E)\right]\right\rangle \\
& =\frac{2}{3} c_{1}\left\{\frac{\pi r^{2}}{\operatorname{Vol}(F)} \chi-\left(\frac{\pi r^{2}}{\operatorname{Vol}(F)}\right)^{2} c_{1}^{2}\right\}+\frac{1}{3} c_{1}-\varepsilon+4 \sum_{j=1}^{n} s\left(\beta_{j}, \gamma_{j} ; \alpha_{j}\right) .
\end{aligned}
$$




\section{Application to geometric Seifert fibred 3-Manifolds}

This section is devoted to providing some explicit formulae for the $\eta$ invariant of geometric Seifert fibred 3-manifolds. We refer to [11] and [13] for basic material on Seifert fibred 3-manifolds and their relevant geometries.

A Seifert fibred 3-manifold can be viewed as an $S^{1}$-fibration $M \longrightarrow F$ over a closed 2-orbifold $F$.

Associated to $M$ is the Seifert invariant $\left(g ;\left(\alpha_{1}, \beta_{1}\right), \cdots,\left(\alpha_{n}, \beta_{n}\right)\right)$.

The Euler number of the Seifert fibration is

$$
e(M \longrightarrow F)=-\sum_{j=1}^{n} \frac{\beta_{j}}{\alpha_{j}} .
$$

The Euler characteristic of the base orbifold $F$ is

$$
\chi=2-2 g-\sum_{j=1}^{n} \frac{\alpha_{j}-1}{\alpha_{j}} .
$$

The relevant geometry of a Seifert fibred 3-manifold is determined by $e$ and $\chi$ as follows.

\begin{tabular}{|l|l|l|l|}
\hline & $\chi>0$ & $\chi=0$ & $\chi<0$ \\
\hline$e=0$ & $\mathbf{S}^{2} \times \mathbf{E}^{1}$ & $\mathbf{E}^{3}$ & $\mathbf{H}^{2} \times \mathbf{E}^{1}$ \\
\hline$e \neq 0$ & $\mathbf{S}^{3}$ & Nil & $\widetilde{\mathbf{P S L}}$ \\
\hline
\end{tabular}

In what follows, we assume that the base space $F$ is oriented.

Let $\boldsymbol{M}=\boldsymbol{M}\left(g ;\left(\alpha_{1}, \beta_{1}\right), \cdots,\left(\alpha_{n}, \beta_{n}\right)\right) \longrightarrow F$ be a geometric Seifert fibred 3-manifold and $E \longrightarrow F$ be the associated $C^{1}$-bundle. Then the set of singular points of the orbifold $E$ is given by $\Sigma=\left\{\left(x_{1}, 0\right), \cdots,\left(x_{n}, 0\right)\right\}$. The local group $G_{x_{j}}=\mathbf{Z} / \alpha_{j}$ acts on the first and the second coordinate by multiplication by $e^{2 \pi \beta_{j} i / \alpha_{j}}$ and $e^{2 \pi i / \alpha_{j}}$ respectively.

Thus, in the expression of $\eta(M)$ in Theorem 2.4,

$$
s\left(\beta_{j}, \gamma_{j} ; \alpha_{j}\right)=s\left(\beta_{j}, 1 ; \alpha_{j}\right)=s\left(\beta_{j}, \alpha_{j}\right) .
$$

Choose a fiber metric $\tilde{g}$ on $E$ such that the induced metric on $M$ is the one from the corresponding Seifert geometry. Let $\tilde{\nabla}$ be a $\tilde{g}$-preserving connection on $E$.

Lemma 3.1. If $M$ is locally symmetric, then under the above assumption, $\tilde{R}$ is constant on $F$.

Proof. Choose the same local basis $\left\{e_{1}=\frac{\partial}{r \partial \theta}, e_{2}=\frac{\partial}{\partial r}, e_{3}, e_{4}\right\}$ as in Section 2. For every $x \in M$, denote $I_{x}$ the local reflection about $x$. Since $M=S_{r}(E)$ is locally symmetric, $I_{x}$ is an isometry. Hence $d I_{x}=-I d$ commutes with $\nabla R$. Thus

$$
\begin{aligned}
& -\nabla_{e_{\alpha}} R\left(e_{3}, e_{4}\right) e_{1}=d I_{x}\left(\nabla_{e_{\alpha}} R\left(e_{3}, e_{4}\right) e_{1}\right) \\
& \quad=\nabla_{-e_{\alpha}} R\left(-e_{3},-e_{4}\right)\left(-e_{1}\right)=\nabla_{e_{\alpha}} R\left(e_{3}, e_{4}\right) e_{1} \text { for } \alpha=3,4 .
\end{aligned}
$$


Therefore,

$$
\nabla_{e_{\alpha}} R\left(e_{3}, e_{4}\right) e_{1}=0 \text { for } \alpha=3,4 .
$$

From Lemma 2.2, we have $\omega_{3}^{2}=\omega_{4}^{2}=0$. Thus,

$$
\nabla_{e_{\alpha}} e_{2}=0 \text { for } \alpha=3,4
$$

It follows that

$$
\begin{aligned}
e_{\alpha}\left(R_{1234}\right) & =e_{\alpha}\left(g\left(R\left(e_{3}, e_{4}\right) e_{1}, e_{2}\right)\right) \\
& =g\left(\nabla_{e_{\alpha}} R\left(e_{3}, e_{4}\right) e_{1}, e_{2}\right)+g\left(R\left(e_{3}, e_{4}\right) e_{1}, \nabla_{e_{\alpha}} e_{2}\right) \\
& =0 \quad \text { for } \alpha=3,4 .
\end{aligned}
$$

Also, from formula (2) in Section 2, we have

$$
\tilde{R} \circ \pi=\tilde{R}_{1234} \circ \pi=R_{1234} .
$$

Thus we get $x_{\alpha}(\tilde{R})=e_{\alpha}(R)=0$ for $\alpha=3,4$. Hence, $\tilde{R}$ is constant on $F$.

Now, we are in position to apply Theorem 2.4 to the geometric Seifert fibred 3-manifolds.

(a) $M$ is modeled on $\mathbf{S}^{2} \times \mathbf{E}^{1}-, \mathbf{E}^{3}$ - or $\mathbf{H}^{2} \times \mathbf{E}^{1}$-geometry.

Any Seifert manifold $M\left(g ;\left(\alpha_{1}, \beta_{1}\right), \cdots,\left(\alpha_{n}, \beta_{n}\right)\right)$ with one of these geometries is locally symmetric and $c_{1}=e=0$. Thus we have by Theorem 2.4 ,

$$
\eta(M)=4 \sum_{j=1}^{n} s\left(\beta_{j}, \alpha_{j}\right)
$$

(b) $M$ is modeled on $\mathbf{S}^{3}$-geometry.

Clearly, every Seifert manifold with this geometry is locally symmetric.

(1) $n \geq 3$. F has an $\mathbf{S}^{2}$-geometry. We have

$$
2 \pi \chi \cdot 2 \pi r=\operatorname{Vol}(M)=\frac{\operatorname{Vol}\left(S^{3}(2)\right)}{\left|\pi_{1}(M)\right|}=\frac{16 \pi^{2}}{4\left|\frac{e}{\chi^{2}}\right|}=4 \pi^{2} \frac{\chi^{2}}{|e|} .
$$

Hence, $r=|\chi / e|$. Thus Theorem 2.4 yields

$$
\eta(M)=\frac{1}{6} \frac{\chi^{2}}{e}+\frac{1}{3} e-\operatorname{sgn}(e)+4 \sum_{j=1}^{n} s\left(\beta_{j}, \alpha_{j}\right) .
$$

(2) $n \leq 2$. We have $M\left(\left(\alpha_{1}, \beta_{1}\right),\left(\alpha_{2}, \beta_{2}\right)\right)=L(p, q)$ where

$$
\epsilon p=\alpha_{1} \beta_{2}+\alpha_{2} \beta_{1}, \quad \epsilon q=\alpha_{1} \beta_{2}^{\prime}+\alpha_{2}^{\prime} \beta_{1}, \quad 1=\alpha_{2} \beta_{2}^{\prime}-\alpha_{2}^{\prime} \beta_{2},
$$

and $\epsilon= \pm 1$.

In order to use Theorem 2.4, we need a "geometric fibration" $M \longrightarrow F$ such that $F$ possesses a geometry from $\mathbf{S}^{2}$ as a 2 -orbifold. This is equivalent to requiring that $\alpha_{1}=\alpha_{2}$. 
Lemma 3.2. Any lens space $L(p, q)$ possesses exactly two geometric Seifert fibrations

$$
L(p, q)=M\left(\left(\alpha, \gamma_{1}\right),\left(\alpha, \gamma_{2}\right)\right)
$$

where

$$
\begin{gathered}
\alpha=\frac{p}{\operatorname{gcd}(p, q-1)}, \quad \gamma_{1}+\gamma_{2}=\operatorname{gcd}(p, q-1), \quad \text { and } \\
\gamma_{2} \frac{q-1}{\operatorname{gcd}(p, q-1)} \equiv-1 \quad(\bmod \alpha)
\end{gathered}
$$

or

$$
L(p, q)=M\left(\left(\alpha^{\prime}, \gamma_{1}^{\prime}\right),\left(\alpha^{\prime}, \gamma_{2}^{\prime}\right)\right)
$$

where

$$
\begin{gathered}
\alpha^{\prime}=\frac{p}{\operatorname{gcd}(p, q+1)}, \quad \gamma_{1}^{\prime}+\gamma_{2}^{\prime}=-\operatorname{gcd}(p, q+1), \quad \text { and } \\
\gamma_{2}^{\prime} \frac{q+1}{\operatorname{gcd}(p, q+1)} \equiv-1 \quad\left(\bmod \alpha^{\prime}\right) .
\end{gathered}
$$

Proof. We will show it in one case. The other case is similar.

Suppose that $\alpha, \gamma_{1}, \gamma_{2}$ satisfies

$$
p=\alpha \gamma_{2}+\alpha \gamma_{1}, \quad q=\alpha \beta_{2}^{\prime}+\alpha_{2}^{\prime} \gamma_{1}, \quad 1=\alpha \beta_{2}^{\prime}-\alpha_{2}^{\prime} \gamma_{2} .
$$

Then we have

$$
1=\alpha \beta_{2}^{\prime}-\frac{q-1}{\gamma_{1}+\gamma_{2}} \gamma_{2}=\frac{p}{\gamma_{1}+\gamma_{2}} \beta_{2}^{\prime}-\frac{q-1}{\gamma_{1}+\gamma_{2}} \gamma_{2} .
$$

It follows that

$$
\begin{gathered}
\gamma_{1}+\gamma_{2}=\operatorname{gcd}(p, q-1), \quad \gamma_{2} \frac{q-1}{\operatorname{gcd}(p, q-1)} \equiv-1 \quad(\bmod \alpha), \quad \text { and } \\
\alpha=\frac{p}{\operatorname{gcd}(p, q-1)} .
\end{gathered}
$$

The converse is straightforward.

For the geometric fibration $L(p, q)=M\left(\left(\alpha, \gamma_{1}\right),\left(\alpha, \gamma_{2}\right)\right)$, we have

$$
e=-\frac{\gamma_{1}+\gamma_{2}}{\alpha}=-\frac{(\operatorname{gcd}(p, q-1))^{2}}{p} \text { and } \chi=2-2 \frac{\alpha-1}{\alpha}=2 \frac{\operatorname{gcd}(p, q-1)}{p} \text {. }
$$

Thus, from Theorem 2.4, we get

$$
\begin{aligned}
\eta(L(p, q)) & =\eta\left(M\left(\left(\alpha, \gamma_{1}\right),\left(\alpha, \gamma_{2}\right)\right)\right) \\
& =\frac{1}{6} \frac{\chi^{2}}{e}+\frac{1}{3} e+\operatorname{sgn}(p)+4 \sum_{j=1}^{2} s\left(\gamma_{j}, \alpha\right) \\
& =-\frac{2}{3 p}-\frac{1}{3} \frac{(\operatorname{gcd}(p, q-1))^{2}}{p}+\operatorname{sgn}(p)+4 \sum_{j=1}^{2} s\left(\gamma_{j}, \alpha\right) .
\end{aligned}
$$

where

$$
\alpha=\frac{p}{\operatorname{gcd}(p, q-1)}, \quad \gamma_{1}+\gamma_{2}=\operatorname{gcd}(p, q-1), \quad \text { and }
$$




$$
\gamma_{2} \frac{q-1}{\operatorname{gcd}(p, q-1)} \equiv-1 \quad(\bmod \alpha)
$$

Similar computation yields

$$
\begin{aligned}
\eta(L(p, q)) & =\eta\left(M\left(\left(\alpha^{\prime}, \gamma_{1}^{\prime}\right),\left(\alpha^{\prime}, \gamma_{2}^{\prime}\right)\right)\right) \\
& =\frac{2}{3 p}+\frac{1}{3} \frac{(\operatorname{gcd}(p, q+1))^{2}}{p}-\operatorname{sgn}(p)+4 \sum_{j=1}^{2} s\left(\gamma_{j}^{\prime}, \alpha^{\prime}\right) .
\end{aligned}
$$

where

$$
\begin{gathered}
\alpha^{\prime}=\frac{p}{\operatorname{gcd}(p, q+1)}, \quad \gamma_{1}^{\prime}+\gamma_{2}^{\prime}=-\operatorname{gcd}(p, q+1), \quad \text { and } \\
\gamma_{2}^{\prime} \frac{q+1}{\operatorname{gcd}(p, q+1)} \equiv-1 \quad\left(\bmod \alpha^{\prime}\right) .
\end{gathered}
$$

On the other hand, as computed by Atiyah-Patodi-Singer in [2]

$$
\eta(L(p, q))=-4 s(q, p) .
$$

Thus, by equating the above three formulae, we get the following interesting identities about the Dedekind sums:

$$
\begin{aligned}
s(q, p) & =-\sum_{j=1}^{2} s\left(\gamma_{j}, \alpha\right)+\frac{1}{6 p}+\frac{1}{12} \frac{(\operatorname{gcd}(p, q-1))^{2}}{p}-\frac{1}{4} \operatorname{sgn}(p) \\
& =-\sum_{j=1}^{2} s\left(\gamma_{j}^{\prime}, \alpha^{\prime}\right)-\frac{1}{6 p}-\frac{1}{12} \frac{(\operatorname{gcd}(p, q+1))^{2}}{p}+\frac{1}{4} \operatorname{sgn}(p)
\end{aligned}
$$

where

$$
\begin{gathered}
\alpha=\frac{p}{\operatorname{gcd}(p, q-1)}, \gamma_{1}+\gamma_{2}=\operatorname{gcd}(p, q-1), \text { and } \\
\gamma_{2} \frac{q-1}{\operatorname{gcd}(p, q-1)} \equiv-1 \quad(\bmod \alpha)
\end{gathered}
$$

and

$$
\begin{gathered}
\alpha^{\prime}=\frac{p}{\operatorname{gcd}(p, q+1)}, \quad \gamma_{1}^{\prime}+\gamma_{2}^{\prime}=-\operatorname{gcd}(p, q+1), \text { and } \\
\gamma_{2}^{\prime} \frac{q+1}{\operatorname{gcd}(p, q+1)} \equiv-1 \quad\left(\bmod \alpha^{\prime}\right) .
\end{gathered}
$$

In particular, let $q=1$ and $p>0$ in the first equality or $q=-1$ and $p<0$ in the second equation, we get

$$
s(1, p)=\frac{1}{12 p}(p-\operatorname{sgn}(p))(p-2 \operatorname{sgn}(p)) .
$$

Thus, we have

$$
\sum_{k=1}^{|p|-1}\left(\cot \left(\frac{\pi k}{p}\right)\right)^{2}=\frac{1}{3}(p-\operatorname{sgn}(p))(p-2 \operatorname{sgn}(p)) .
$$

(c) $M$ is modeled on $\widetilde{P S L}$-geometry. Equip $\mathbf{H}^{2}$ with the standard hyperbolic metric. Then we have a natural metric on $T\left(\mathbf{H}^{2}\right)$. The identification 
between $P S L(2, \mathbf{R})$ and the unit tangent bundle $T^{1}\left(\mathbf{H}^{2}\right)$ gives rise to a (leftinvariant) metric on $\operatorname{PSL}(2, \mathbf{R})$ which induces a metric on $\widetilde{P S L}$.

For a Seifert manifold $M \longrightarrow F$ with this geometry and the given metric on $\widetilde{P S L}$, we have $\tilde{R}=-1$ on $F$. Thus, Theorem 2.4 implies.

From a homomorphism $\pi_{1}(M) \longrightarrow \operatorname{Isom}(\widetilde{P S L})$ giving a geometric structure on $M$, we get $r=|\chi / e|$ (see [11]). It follows from Theorem 2.4 that

$$
\eta(M)=-\frac{1}{2} \frac{\chi^{2}}{e}+\frac{1}{3} e-\operatorname{sgn}(e)+4 \sum_{j=1}^{n} s\left(\beta_{j}, \alpha_{j}\right) .
$$

From the above discussion, we have the following

Corollary 3.3. Under the above five geometries, if we fix the metric in each universal cover as above, then the n-invariant depends only on the topology.

Finally, under the Nil-geometry, the volume of the base orbifold is indeterminate, so the Seifert invariant alone is not sufficient to express $\eta(M)$.

We conclude this section with the following

Corollary 3.4. Equip $\operatorname{PSL}(2, \mathbf{R})$ with the above metric. Let $\Gamma \subseteq P S L(2, \mathbf{R})$ be a co-compact Fuchsian group of signature $\left\{g ; \alpha_{1}, \cdots, \alpha_{n}\right\}$. Then

$$
\eta(P S L(2, \mathbf{R}) / \Gamma)=\frac{1}{6}(2 g+4+7 n)-\sum_{j=1}^{n}\left(\frac{1}{3} \alpha_{j}+\frac{5}{6 \alpha_{j}}\right) .
$$

Proof. As shown in [11],

$$
\operatorname{PSL}(2, \mathbf{R}) / \Gamma=M\left(g ;(1,2 g-2),\left(\alpha_{1}, \alpha_{1}-1\right), \cdots,\left(\alpha_{n}, \alpha_{n}-1\right)\right) .
$$

Thus,

$$
\chi=e=2-2 g-\sum_{j=1}^{n} \frac{\alpha_{j}-1}{\alpha_{j}} .
$$

Also, from (3.1), we get

$$
s\left(\alpha_{j}-1 ; \alpha_{j}\right)=-s\left(1, \alpha_{j}\right)=-\frac{1}{12 \alpha_{j}}\left(\alpha_{j}-1\right)\left(\alpha_{j}-2\right) .
$$

It follows from (3.2) that

$$
\begin{aligned}
\eta(P S L(2, \mathbf{R}) / \Gamma) & =-\frac{1}{2} \chi+\frac{1}{3} \chi+1+4 \sum_{j=1}^{n} s\left(\alpha_{j}-1, \alpha_{j}\right) \\
& =-\frac{1}{6} \chi+1+4 \sum_{j=1}^{n}\left(-\frac{1}{12 \alpha_{j}}\left(\alpha_{j}-1\right)\left(\alpha_{j}-2\right)\right) \\
& =\frac{1}{6}(2 g+4+7 n)-\sum_{j=1}^{n}\left(\frac{1}{3} \alpha_{j}+\frac{5}{6 \alpha_{j}}\right) \cdot
\end{aligned}
$$

Remark 1. A similar formula for the the $\eta$-invariant of $P S L(2, \mathbf{R}) / \Gamma$ associated to the Dirac operator was obtained in [14]. 


\section{Chern-Simons INVARIANT AND CONFORMAL IMMERSIONS}

Let $M$ be a closed, oriented Riemannian 3-manifold. Chern and Simons defined a mod 1 invariant of $M$ in [3], now commonly denoted by $C S(M)$, and showed that $C S(M) \equiv 0(\bmod 1)$ if $M$ conformally immerses into $\mathbf{E}^{4}$.

A surprising relation between $C S(M)$ and $\eta(M)$ is demonstrated by the following

Theorem 4.1 (Atiyah-Patodi-Singer [2]). Let $M$ be a closed, oriented Riemannian 3-manifold. Then

$$
C S(M) \equiv \frac{3}{2} \eta(M)+\frac{1}{2} \sigma\left(H_{1}(M ; Z)\right)(\bmod 1)
$$

where $\sigma\left(H_{1}(M ; \mathbf{Z})\right)=$ \# of 2-primary summands in $H_{1}(M ; \mathbf{Z})$.

In this section, we derive some elementary formulae for the Chern-Simons invariant of the geometric Seifert fibred 3-manifolds and show that some families of them cannot be conformally immersed into $\mathbf{E}^{4}$. We begin with the following

Lemma 4.2. Let $p, q$ be a pair of coprime positive integers. Choose $r, s$ such that $p s+q r=1, q+r$ is even and $s$ is odd if $p$ is even. Then

$$
6 s(q, p) \equiv \frac{q+r}{2 p}(\bmod 1) .
$$

Proof. Case 1. $p$ is odd. We have

$$
6 p s(q, p)=\frac{q+r_{0}}{2}+\frac{1}{2} p I(p, q) \in \mathbf{Z}
$$

where $r_{0}$ is such that $q r_{0} \equiv 1(\bmod p),-1<r_{0} / p \leq 0$, and $I(p, q) \in \mathbf{Z}$ (see [7], for instance). Since $p$ is odd, $q+r_{0}$ and $I(p, q)$ have the same parity. It follows that

$$
6 s(q, p) \equiv \frac{q+r_{0}+\delta p}{2 p} \quad(\bmod 1)
$$

with

$$
\delta= \begin{cases}0 & \text { if } q+r_{0} \text { is even } \\ 1 & \text { if } q+r_{0} \text { is odd }\end{cases}
$$

Therefore we have

$$
6 s(q, p) \equiv \frac{q+r}{2 p}(\bmod 1)
$$

with $q r \equiv 1(\bmod p)$ and $q+r$ even. 
Case 2. $p$ is even. Then $q$ is odd.

From the Dedekind reciprocity law and Case 1, we get

$$
\begin{aligned}
6 s(q, p) & =-6 s(p, q)+\frac{p^{2}+q^{2}+1-3 p q}{2 p q} \\
& \equiv \frac{-p-t}{2 q}+\frac{p^{2}+q^{2}+1-3 p q}{2 p q}(\bmod 1), \quad p t \equiv 1(\bmod q), t \text { even } \\
& \equiv \frac{q^{2}+1-p s}{2 p q} \quad(\bmod 1), \quad p s \equiv 1(\bmod q), s \text { odd } \\
& \equiv \frac{q+r}{2 p}(\bmod 1), \quad p s+q r=1, s \text { odd. }
\end{aligned}
$$

Lemma 4.3. Let

$$
M=M\left(g ;\left(\alpha_{1}, \beta_{1}\right), \cdots,\left(\alpha_{n}, \beta_{n}\right)\right.
$$

be a Seifert fibred 3-manifold and l the number of even $\alpha_{j}$ 's. Then

$$
\sigma\left(H_{1}(M ; \mathbf{Z})\right)= \begin{cases}l-1 & \text { if } l \geq 1, \\ 1 & \text { if } l=0 \text { and } \sum \beta_{j} \text { is even, } \\ 0 & \text { if } l=0 \text { and } \sum \beta_{j} \text { is odd. }\end{cases}
$$

Proof. Arrange the Seifert invariant such that

$$
M=M\left(g ;(1, a),\left(\alpha_{1}, \beta_{1}\right), \cdots,\left(\alpha_{l}, \beta_{l}\right),\left(\alpha_{l+1}, \beta_{l+1}^{\prime}\right), \cdots,\left(\alpha_{n}, \beta_{n}^{\prime}\right)\right)
$$

where $\alpha_{j}$ is even for $j \leq l, \alpha_{j}$ is odd for $j>l, \beta_{j}^{\prime}$ is even for $j>l$, and $a$ is the number of odd $\beta_{j}$ 's for $j>l$. Then we have

$$
H_{1}(M ; \mathbf{Z})=\mathbf{Z}^{2 g} \oplus \operatorname{Cok}\left(\left(\begin{array}{cccccccc}
1 & 1 & \cdots & 1 & 1 & \cdots & 1 & 0 \\
1 & 0 & \cdots & 0 & 0 & \cdots & 0 & a \\
& \alpha_{1} & & & & & & \beta_{1} \\
& & \ddots & & & & & \vdots \\
& & & \alpha_{l} & & & & \beta_{l} \\
& & & & \alpha_{l+1} & & & \beta_{l+1} \\
& & & & & \ddots & & \vdots \\
& & & & & & \alpha_{n} & \beta_{n}
\end{array}\right)\right. \text {. }
$$


Therefore

$$
\begin{aligned}
& \sigma\left(H_{1}(M ; \mathbf{Z})\right)=\sigma\left(\operatorname{Cok}\left(\left(\begin{array}{cccccccc}
1 & 1 & \cdots & 1 & 1 & \cdots & 1 & 0 \\
1 & 0 & \cdots & 0 & 0 & \cdots & 0 & a \\
& \alpha_{1} & & & & & & \beta_{1} \\
& & \ddots & & & & & \vdots \\
& & & \alpha_{l} & & & & \beta_{l} \\
& & & & \alpha_{l+1} & & & \beta_{l+1} \\
& & & & & \ddots & & \vdots \\
& & & & & & \alpha_{n} & \beta_{n}
\end{array}\right)\right)\right. \\
& =\sigma\left(\operatorname{Cok}\left(\left(\begin{array}{cccccccc}
1 & 1 & \cdots & 1 & 1 & \cdots & 1 & 0 \\
1 & 0 & \cdots & 0 & 0 & \cdots & 0 & a \\
& 0 & & & & & & 1 \\
& & \ddots & & & & & \vdots \\
& & & 0 & & & & 1 \\
& & & & 1 & & & 0 \\
& & & & & \ddots & & \vdots \\
& & & & & & 1 & 0
\end{array}\right)\right)\right. \text {. }
\end{aligned}
$$

It follows that

$$
\begin{aligned}
\sigma\left(H_{1}(M ; \mathbf{Z})\right) & = \begin{cases}l-1 & \text { if } l \geq 1, \\
1 & \text { if } l=0 \text { and } a \text { is even, } \\
0 & \text { if } l=0 \text { and } a \text { is odd, }\end{cases} \\
& = \begin{cases}l-1 & \text { if } l \geq 1, \\
1 & \text { if } l=0 \text { and } \sum \beta_{j} \text { is even, } \\
0 & \text { if } l=0 \text { and } \sum \beta_{j} \text { is odd. }\end{cases}
\end{aligned}
$$

By virtue of the formulae for $\eta(M)$ in Section 3, Theorem 4.1, Lemma 4.2 and Lemma 4.3, we derive the following elementary formulae for the ChernSimons invariant of the geometric Seifert fibred 3-manifolds.

Without loss of generality, we assume in what follows that our Seifert invariant is in a normal form $\left((1, b),\left(\alpha_{1}, \beta_{1}\right), \cdots,\left(\alpha_{n}, \beta_{n}\right)\right)$. For every pair of $\left(\alpha_{j}, \beta_{j}\right)$, choose $r_{j}, s_{j}$ such that $\alpha_{j} s_{j}+\beta_{j} r_{j}=1, \beta_{j}+r_{j}$ is even and $s_{j}$ is odd if $\alpha_{j}$ is even.

(a) $M$ is modeled on $\mathbf{E}^{3}, \mathbf{S}^{2} \times \mathbf{E}^{1}$, or $\mathbf{H}^{2} \times \mathbf{E}^{1}$-geometry. We have

$$
\begin{aligned}
C S(M) & \equiv 6 \sum_{j=1}^{n} s\left(\beta_{j}, \alpha_{j}\right)+\frac{1}{2} \sigma\left(H_{1}(M ; \mathbf{Z})\right) \quad(\bmod 1) \\
& \equiv\left\{\begin{array}{l}
\sum_{j=1}^{n} r_{j} /\left(2 \alpha_{j}\right)+(l-1) / 2(\bmod 1) \text { if } l \geq 1, \\
\sum_{j=1}^{n} r_{j} /\left(2 \alpha_{j}\right)+1 / 2(\bmod 1) \text { if } l=0 \text { and } b+\sum \beta_{j} \text { is even, } \\
\sum_{j=1}^{n} r_{j} /\left(2 \alpha_{j}\right)(\bmod 1) \text { if } l=0 \text { and } b+\sum \beta_{j} \text { is odd. }
\end{array}\right.
\end{aligned}
$$


(b) $M$ is modeled on $\mathbf{S}^{3}$-geometry.

(i) $n \geq 3$. We have

$$
C S(M) \equiv \frac{1}{4} \frac{\chi^{2}}{e}+\frac{1}{2} e+\frac{1}{2}+6 \sum_{j=1}^{n} s\left(\beta_{j}, \alpha_{j}\right)+\frac{1}{2} \sigma\left(H_{1}(M ; \mathbf{Z})\right) \quad(\bmod 1) .
$$

Thus,

$$
\begin{aligned}
C S(M) & \equiv \frac{1}{4} \frac{\chi^{2}}{e}+\sum_{j=1}^{n} \frac{r_{j}}{2 \alpha_{j}}+\frac{1}{2} l(\bmod 1) \quad \text { if } l \geq 1 \\
& \equiv \frac{\chi^{2}}{4 e}+\sum_{j=1}^{n} \frac{r_{j}}{2 \alpha_{j}}(\bmod 1) \text { if } l=0 \text { and } b+\sum \beta_{j} \text { is even } \\
& \equiv \frac{\chi^{2}}{4 e}+\frac{1}{2}+\sum_{j=1}^{n} \frac{r_{j}}{2 \alpha_{j}}(\bmod 1) \text { if } l=0 \text { and } b+\sum \beta_{j} \text { is odd. }
\end{aligned}
$$

(ii) $n \leq 2$. We have $M\left(\left(\alpha_{1}, \beta_{1}\right),\left(\alpha_{2}, \beta_{2}\right)\right)=L(p, q)$ and

$$
\begin{aligned}
C S(L(p, q)) & \equiv-6 s(q, p)+\frac{1}{2} \delta(L(p, q))(\bmod 1) \\
& \equiv-\frac{q+r}{2 p} \quad(\bmod 1)
\end{aligned}
$$

where $p s+q r=1$ with $q+r$ even and $s$ even if $p$ is even.

(c) $M$ is modeled on PSL-geometry.

$$
C S(M) \equiv-\frac{3}{4} \frac{\chi^{2}}{e}+\frac{1}{2} e+\frac{1}{2}+6 \sum_{j=1}^{n} s\left(\beta_{j}, \alpha_{j}\right)+\frac{1}{2} \sigma\left(H_{1}(M ; Z)\right) \quad(\bmod 1) .
$$

Thus,

$$
C S(M) \equiv-\frac{3}{4} \frac{\chi^{2}}{e}+\sum_{j=1}^{n} \frac{r_{j}}{2 \alpha_{j}}+\frac{1}{2} l(\bmod 1) \quad \text { if } l \geq 1,
$$

$$
\begin{aligned}
& \equiv-\frac{3 \chi^{2}}{4 e}+\sum_{j=1}^{n} \frac{r_{j}}{2 \alpha_{j}}(\bmod 1) \text { if } l=0 \text { and } b+\sum \beta_{j} \text { is even, } \\
& \equiv-\frac{3 \chi^{2}}{4 e}+\frac{1}{2}+\sum_{j=1}^{n} \frac{r_{j}}{2 \alpha_{j}}(\bmod 1) \text { if } l=0 \text { and } b+\sum \beta_{j} \text { is odd. }
\end{aligned}
$$

As Hirsch showed in [6], all compact 3-manifolds immerse in $\mathbf{R}^{4}$. We will show that some families of the geometric Seifert fibred 3-manifolds cannot be conformally immersed into $\mathbf{E}^{4}$.

Corollary 4.4. Let $F_{g}$ be the surface of genus $g>1$ with a hyperbolic geometry. If $g$ is even, then $T^{1}\left(F_{g}\right)=M(g,(1,2 g-2))$ with the induced metric from $\mathbf{H}^{2}$ doesn't conformally immerse into $\mathbf{E}^{4}$. 
Proof. We have $e=\chi=2-2 g$. It follows from (4.2) that

$$
C S\left(T^{1}\left(F_{g}\right)\right) \equiv \frac{g}{2}-\frac{1}{2}(\bmod 1) \neq 0 \text { if } g \text { is even. }
$$

Corollary 4.5. Equip $S O(3)$ with a bi-invariant metric. Let $\Gamma$ be a finite subgroup of $S O(3)$. Then $S O(3) / \Gamma$ doesn't conformally immerse into $\mathbf{E}^{4}$.

Proof. By the conformal invariance of the Chern-Simons invariant, we can assume that $S O(3)$ possesses the standard metric from $\mathbf{S}^{3}(1)$.

From (5) we have

$$
C S(S O(3))=C S(L(2,1)) \equiv-\frac{1}{2}(\bmod 1) .
$$

Thus, $S O(3)$ cannot be conformally immersed into $\mathrm{E}^{4}$. Hence $S O(3) / \Gamma$ cannot be conformally immersed into $\mathbf{E}^{4}$.

Remark. Heitsch and Lawson in [5] showed that a similar result holds in general for $S O(2 k+1) / \Gamma$ where $\Gamma$ is a discrete subgroup of $S O(2 k+1)$ and $S O(2 k+$ $1)$ is equipped with a bi-invariant metric.

Corollary 4.6. $L(p, q)$ with the standard metric cannot be conformally immersed into $\mathbf{E}^{4}$ except possibly when $q^{2}+1 \equiv 0(\bmod p)$ and $p$ is odd.

Proof. If $p=2 k$ is even, then

$$
L(p, q)=\mathbf{S}^{3} /(\mathbf{Z} / 2 k)=S O(3) /(\mathbf{Z} / k) .
$$

Thus, Corollary 4.5 implies that $L(p, q)$ cannot be conformally immersed into $\mathbf{E}^{4}$;

If $p$ is odd, then from (4.1) we get

$$
C S(L(p, q)) \equiv-\frac{q+r}{2 p} \quad(\bmod 1)
$$

with $q r \equiv 1(\bmod p)$ and $q+r$ even. Thus, $C S(L(p, q)) \equiv 0(\bmod 1)$ implies $q+r \equiv 0(\bmod p)$. Hence $q^{2}+1 \equiv 0(\bmod p)$.

\section{ACKNOWLEDGMENTS}

This paper presents part of my Ph.D. thesis. I am much indebted to my advisor Professor Walter D. Neumann for his guidance. I also thank Professors Michael Davis and Ernst Ruh for some helpful conversations. Finally, I would like to thank the referee for his (her) kind corrections and suggestions.

\section{REFERENCES}

1. M. Atiyah, V. Patodi, and I. Singer, Spectral asymmetry and Riemannian geometry. I, Math. Proc. Cambridge Philos. Soc. 77 (1975), 43-69.

2. Spectral asymmetry and Riemannian geometry. II, Math. Proc. Cambridge Philos. Soc. 78 (1975), 402-433.

3. S. Chern and J. Simons, Characteristic forms and geometric invariants, Ann. of Math. (2) 99 (1974), 48-69.

4. P. Gilkey, The boundary integrand in the formula for the signature and Euler characteristic of a Riemannian manifold with boundary, Adv. Math. 15 (1975), 344-360.

5. J. Heitsch and $\mathrm{H}$. Lawson, Transgressions, Chern-Simons invariants and the classical groups, J. Differential Geom. 9 (1974), 423-434. 
6. M. Hirsch, Immersion of manifolds, Trans. Amer. Math. Soc. 93 (1959), 242-276.

7. F. Hirzebruch and D. Zagier, The Atiyah-Singer theorem and elementary number theory, Publish or Perish, 1974.

8. T. Kawasaki, The signature theorem for $V$-manifolds, Topology 17 (1978), 75-83.

9. The index of elliptic operators over $V$-manifolds, Nagoya Math. J. 84 (1981), 135-157.

10. M. Komuro, On the Atiyah-Padoti-Singer $\eta$-invariant for $S^{1}$-bundles over Riemann surfaces, J. Fac. Sci. Univ. Tokyo Sect. IA Math. 30 (1984), 525-548.

11. W. Neumann and M. Jankins, Seifert manifolds, Lecture notes, Brandeis Univ., 1981.

12. I. Satake, The Gauss-Bonnet theorem for V-manifolds, J. Math. Soc. Japan 9 (1957), 464-493.

13. P. Scott, The geometries of 3-manifolds, Bull. London. Math. Soc. 15 (1983), 401-487.

14. J. Seade and B. Steer, $A$ note on the eta function for quotients of $P S L_{2}(\mathbf{R})$ by co-compact Fuchsian groups, Topology 26 (1987), 79-91.

15. W. Thurston, The geometry and topology of 3-manifolds, Lecture notes, Princeton Univ., 1978.

16. W. Thurston, Three dimensional manifolds, Kleinian groups and hyperbolic geometry, Bull. Amer. Math. Soc. 6 (1982), 357-381.

Department of Mathematics, Ohio State University, Columbus, Ohio 43210 48109

Current address: Department of Mathematics, University of Michigan, Ann Arbor, Michigan

E-mail address: ouyang@math.ohio-state.edu 